\title{
Bilateral atraumatic acute anterior compartment syndrome of the legs with rhabdomyolysis and acute kidney injury
}

\author{
Nalaka Gunawansa ${ }^{1,2}$, Sameera Jayasinghe \\ ${ }^{1}$ National Institute of Nephrology Dialysis and Transplant, Sri Lanka \\ ${ }^{2}$ University of Liverpool, United Kingdom \\ ${ }^{3}$ National Hospital of Sri Lanka
}

Key words: compartment syndrome; fasciotomy;

rhabdomyolysis; acute kidney injury; exercise

\section{Introduction}

Acute Compartment Syndrome (ACS) occurs within closed fascial compartments where the intra-compartmental pressure increases progressively, resulting in reduced tissue perfusion within the compartment [1]. The increase in interstitial pressure eventually overcomes the capillary perfusion pressure, resulting in a state of tissue hypoxia. Once the interstitial pressure rises $>30 \mathrm{mmHg}$ or within $25 \mathrm{mmHg}$ of the diastolic pressure, muscle ischemia begins [2]. Hypoxic injury releases metabolites that trigger further inflammation and further increase in compartmental pressure with progressive tissue injury. While ACS can occur in numerous locations including abdomen, thoracic cavity and brain, the commonest form is seen in the muscular compartments of the limbs, primarily the lower limbs.

Lower limb compartment syndrome can be either acute or chronic. ACS is usually seen after trauma including fractures, crush injury, burns, inadvertent intra-arterial injections or severe infections. It can also occur following reperfusion of an ischaemic limb. The annual incidence of post-traumatic ACS has been reported to be as high as 3 per 100,000 [3]. Chronic compartment syndrome (CCS) is less common, usually seen in young athletes or military personnel after strenuous exercise. Atraumatic post-exertional ACS is seen less frequently, usually unilateral and in an individual with a preceding history of CCS. The occurrence of bilateral atraumatic ACS without a history of CCS is extremely rare with only a handful of reported cases. This report describes such a patient with atraumatic bilateral ACS complicated with rhabdomyolysis and acute kidney injury (AKI).

\section{Case presentation}

A 34-year old man presented to the Emergency Department with a history of painful swelling in both legs for 8 days. He is a computer professional working overseas and had a

Correspondence: Nalaka Gunawansa

E-mail: vascular@drnalakagunawansa.com

Received: 31-10-2017 Accepted: 24-11-2017

(iD) http://orcid.org/0000-0003-0098-3855

DOI: http://doi.org/10.4038/sljs.v35i4.8439

The Sri Lanka Journal of Surgery 2017; 35(4): 39-41 relatively sedentary life style. He had no known comorbidities, was a non-smoker and did not use recreational drugs. He had no preceding history of CCS. One day after work he had done extended jogging and came home with some unusual discomfort in the legs. He tried simple home remedies but the symptoms rapidly deteriorated. At the local hospital, he was treated for suspected cellulitis due to the red discolouration on his legs. His condition worsened with progressive pain, swelling and weakness of the legs. By day03, he noticed dark brown discolouration of his urine. Due to his progressive deterioration, he was brought back to Sri Lanka and immediately admitted to hospital.

On initial assessment, he was febrile (37.4 C) and in severe pain. The pulse rate was $96 / \mathrm{min}$ with a respiratory rate of $20 / \mathrm{min}$. The blood pressure was $110 / 65 \mathrm{mmHg}$. There was marked swelling in both legs below the knee, with a well demarcated reddish discolouration corresponding to the anterior compartments (Figure 1). This region was severely tender and extremely firm compared to the rest of the calf.

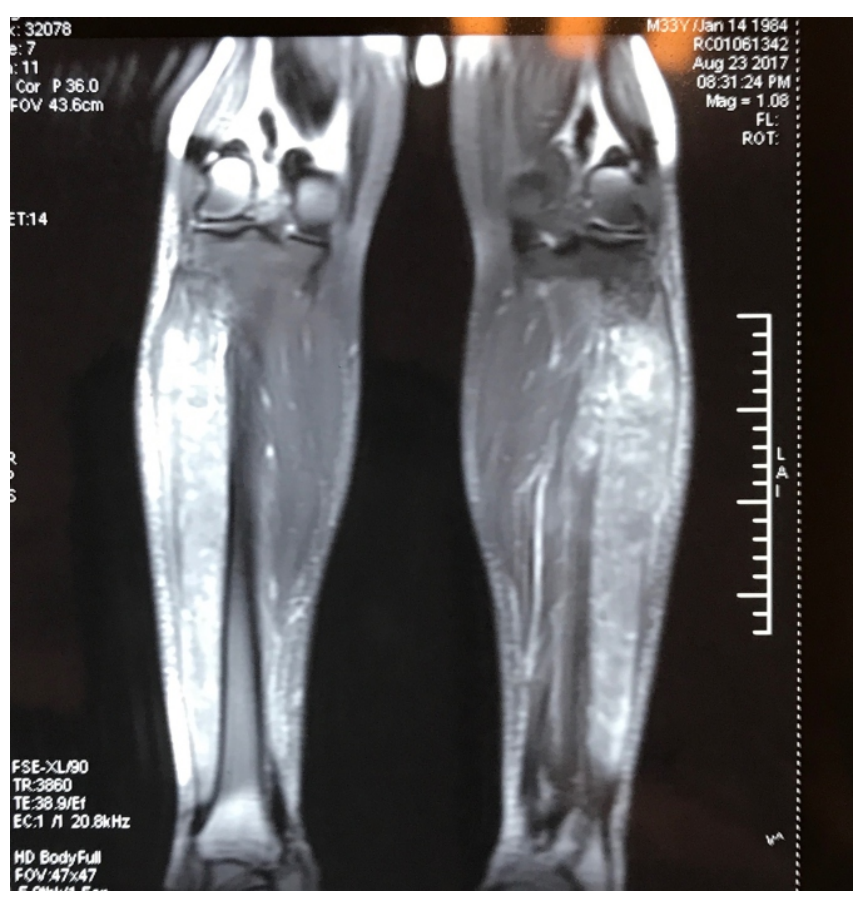

Figure 1. MRI scan showing bilateral anterior compartment oedema and ischaemic damage. Posterior compartments are spared. 
While the posterior tibial pulses were normal, the anterior tibial pulse was not palpable on either side. Doppler assessment showed a triphasic signal over the posterior tibial and a monophasic signal over the anterior tibial artery. He had a complete bilateral foot drop with significant paraesthesia around the dorsum of the foot. He had an indwelling urinary catheter and the urine was dark brown.

Initial blood biochemistry results were; white cell count $19.8 \times 109 / 1$, haemoglobin - $14.7 \mathrm{~g} / \mathrm{dl}$, serum creatinine - 187 $\mathrm{mol} / \mathrm{l}$ and serum creatine phospokinase (CPK) - 100,500 U/1. Urine for myoglobin was strongly positive.

A clinical diagnosis of bilateral ACS with rhabdomyolysis and AKI was made. An urgent MRI scan was done to assess the degree of muscle involvement and showed isolated whitening in bilateral anterior compartments (Figure 2). Compartmental pressure measurements were not pursued due to the overwhelming clinical evidence in favour of the diagnosis.

The condition, therapeutic options and potential outcomes were discussed with the patient and his spouse. Emergency bilateral decompressive fasciotomy was performed, with a full length anterior compartment releasing incision and a limited lateral incision to release the other three compartments. Anterior compartment muscles were nonviable on both legs, being pale brown with no capillary bleeding and no contractility. All other compartments showed

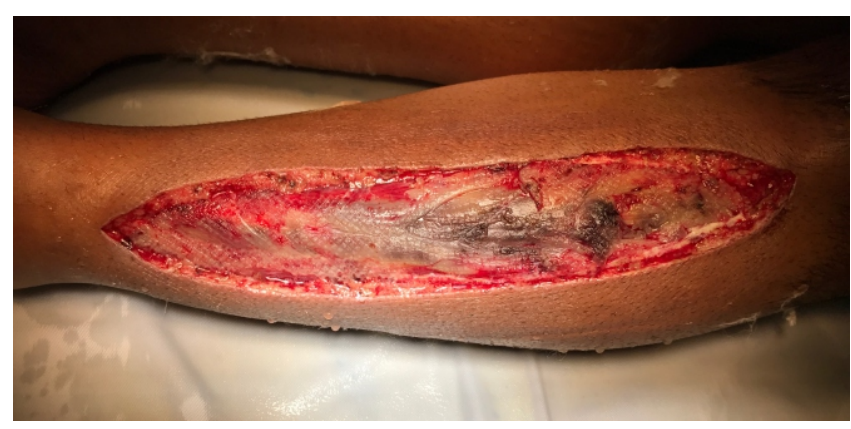

Figure 2. Lateral fasciotomy of the left calf showing

normal viability. The wounds were left open with soft dressings. Prophylactic intravenous antibiotics (meropenam $500 \mathrm{mg} 8$ hourly) were continued peri-operatively. Furthermore, aggressive fluid resuscitation was done along with urinary alkalinization (sodium bicarbonate) and forced diuresis (mannitol) to minimize the effects of AKI.

The immediate post-operative recovery was uncomplicated with dramatic improvement in pain, swelling and degree of paraesthesia. On post-operative day-03, he was afebrile and the urine colour had returned to normal. Urine for myoglobin was negative at this stage. The serum creatinine returned to normal limits by day- 03 . There was dramatic improvement in the serum CPK levels over the next few days (Graph 1). Active physiotherapy was started and he was mobilized after day-03. A negative pressure vacuum system was applied to

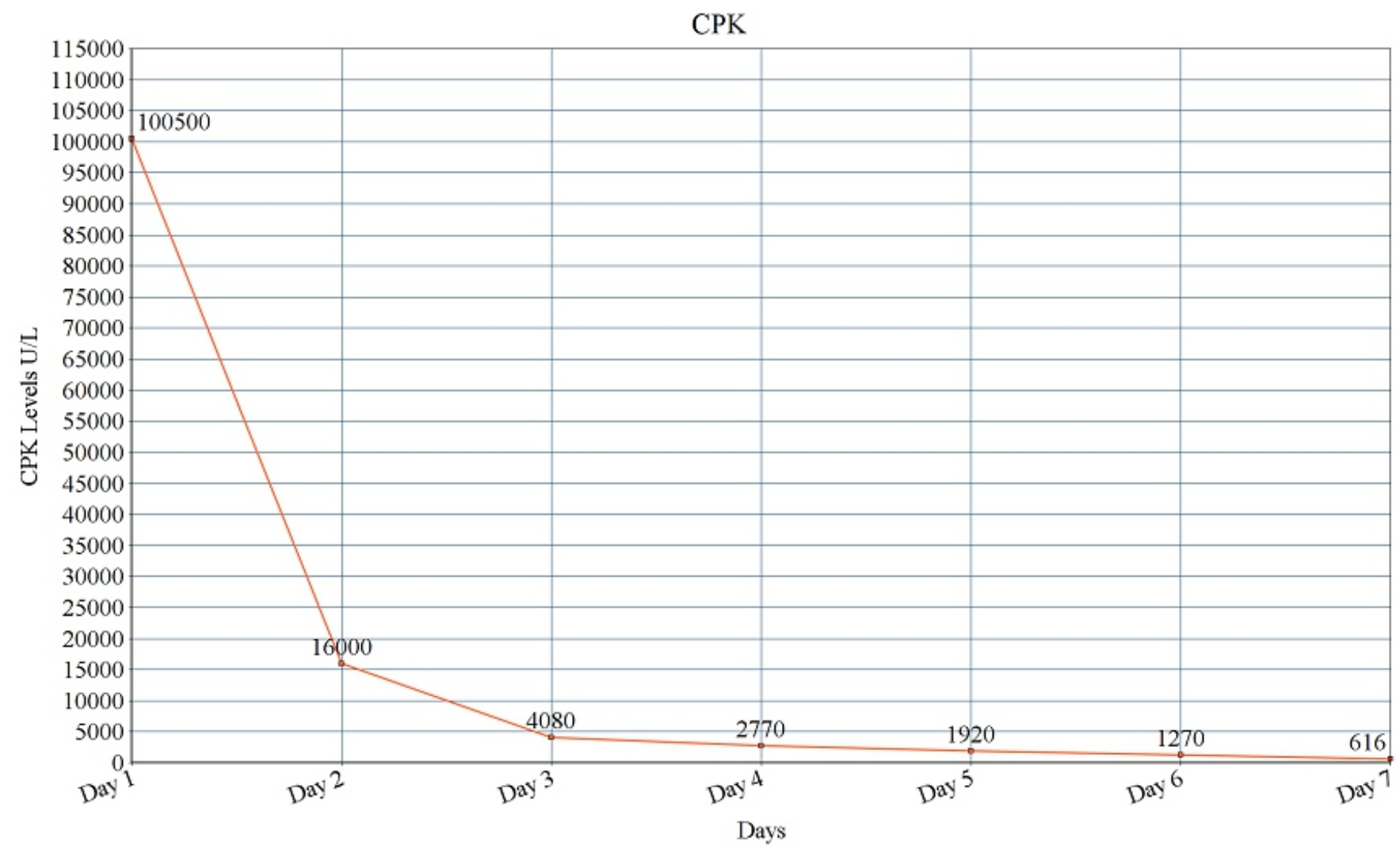

Graph 1. CPK level decline after fasciotomy 
the fasciotomy wounds on day-05. By day-09, he was painfree and able to walk with support. He was discharged at this time with continued physiotherapy and oral antibiotics. The negative pressure dressing was changed at weekly intervals.

Approximately 3 weeks after the fasciotomy, his wounds showed good granulation and no infection. The negative pressure dressing was removed and non-viable tibilais anterior muscle was excised. A split skin grafting was performed and wounds were closed. At 4 weeks follow up, he remains independently mobile. Although some degree of foot drop remains, with sustained physiotherapy he remains minimally disabled and is able to walk and climb stairs independently.

\section{Discussion}

The earliest description of atraumatic exercise induced ACS dates back to 1954, where Dr. Edward Wilson described his own symptoms during an Antarctic expedition [4]. Subsequently, several reports appeared that described exercise induced ACS causing muscle necrosis, usually in soldiers or athletes, termed 'march myositis' [5,6]. However, exercise induced bilateral ACS is extremely rare with only a handful of reported cases [7].

In the absence of a preceding CCS or significant local trauma, the early presentation of ACS can often be misleading, resulting in significant delay in diagnosis. ACS is primarily a clinical diagnosis and a high index of suspicion is required to avoid catastrophic complications. Severe unrelenting pain with tense muscle compartments should always raise the suspicion of ACS. The associated rise in serum CPK is a significant determinant in the early diagnosis [8]. Treatment involves immediate and complete fasciotomy and compartment decompression. Although early stages ACS may be managed conservatively with compartmental pressure monitoring, advanced ACS such as in this patient requires immediate fasciotomy. Supportive care is needed to avoid secondary infection and minimize effects of rhabdomyolysis induced AKI. Once stabilized, early physiotherapy and mobilization can minimize long-term disability. It requires a team approach with surgeons, nephrologists, medical intensivists, physiotherapists and dedicated nursing staff.

\section{References}

1. Elliott KGB, Johnstone AJ. Diagnosing acute compartment syndrome. J Bone Joint Surg Br 2003;85:625-32.

2. Shrier I, Magder S. Pressure-flow relationships in in vitro model of compartment syndrome. J Appl Physiol 1995;79:214-21.

3. McQueen MM, Gaston P, Court-Brown CM. Acute compartment syndrome: Who is at risk? J Bone Jt Surg 1999;82-B:200-3. doi:10.1302/0301-620X.82B2.9799.

4. FREEDMAN BJ. Dr. Edward Wilson of the Antarctic; a biographical sketch, followed by an inquiry into the nature of his last illness. Proc R Soc Med 1954;47:183-9.

5. BLANDY JP, FULLER R. March gangrene; ischaemic myositis of the leg muscle from exercise. $J$ Bone Joint Surg $\mathrm{Br}$ 1957;39-B:679-93.

6. Esmail AN, Flynn JM, Ganley TJ, Pill SG, Harnly H. Acute Exercise-Induced Compartment Syndrome in the Anterior Leg. Am J Sports Med 2001;29:509-12. doi:10.1177/03635465010290042101.

7. McDonald LS, Mitchell RJ, Deaton TG. Bilateral compartment syndrome of the anterior thigh following functional fitness exercises: a case report. Mil Med 2012;177:993-6. doi:10.1136/bcr-2015-210856.

8. Valdez C, Schroeder E, Amdur R, Pascual J, Sarani B. Serum creatine kinase levels are associated with extremity compartment syndrome. J Trauma Acute Care Surg 2013;74:441-7. doi:10.1097/TA.0b013e31827a0a36

\section{Learning Points:}

- Anterior compartment syndrome can occur without a significant history of trauma.

- A high index of suspicion is required in cases of unexplained pain and swelling confined to one compartment.

- Accurate clinical diagnosis is the cornerstone in management.

- Unnecessary delay in diagnosis can have devastating consequences including renal damage, septicaemia and death. 